Published in final edited form as:

Curr Opin HIV AIDS. 2009 January ; 4(1): 11-15. doi:10.1097/COH.0b013e32831a6fc9.

\title{
Stem Cell transplantation in HIV infected patients
}

\author{
Amrita Krishnan, MD, FACP \\ City of Hope National Medical Center, Dept Heme/HCT, 1500 East Duarte Road, Duarte CA \\ 91010, Tel :626 3598111 ext 63974, Fax 6263018973
}

Amrita Krishnan: akrishnan@coh.org

\section{Abstract}

Lymphoma remains a leading cause of mortality in HIV infected patients. In the HIV negative setting high dose therapy with autologous stem cell rescue (ASCT) has been a long accepted treatment for certain malignancies such as lymphoma and leukemia. Early transplant trials excluded older patients, and patients with comorbidities such as HIV infection. However, the procedural related mortality of transplantation has decreased both due to the use of peripheral blood stem cells instead of bone marrow and due to the use of new reduced intensity conditioning regimens. During this same era, the treatment of HIV infection has also become more effective. Patients are no longer dying of opportunistic infections and in addition, their hematologic function has improved.

With these advances in HIV therapy it is possible for HIV infected patients to mobilize adequate numbers of stem cells for an autologous transplant. In addition, with appropriate antiretroviral therapy and infection prophylaxis, the HIV infected patient can tolerate intensive doses of chemotherapy. This review will summarize clinical trials of ASCT in HIV positive patients. Furthermore, the field of solid organ transplantation has grown to also include HIV positive patients. The challenges in solid organ transplantation are similar to allogeneic stem cell transplantation, namely that patients require chronic immunosuppression. This article will also review some of the approaches to allogeneic stem cell transplantation in the HIV positive patient and provide a rationale for the broader use of stem cell transplantation for appropriate HIV related hematologic malignancies.

\section{Keywords}

Autologous transplant; Allogeneic Transplant; Lymphoma

\section{Introduction}

\section{HIV and Malignancy}

With the advent of highly active retroviral therapy (HAART) the complications associated with HIV infection have changed. The incidence of opportunistic infections has decreased and longevity of HIV infected individuals has increased. However, malignancies are now the leading cause of mortality in the individual with HIV infection [1]. It has long been recognized that the incidence of B cell Non Hodgkin Lymphoma (NHL) is increased in HIV infected patients [2]. Moreover other non-AIDS defining malignancies such as Hodgkin lymphoma (HL) are also increased in incidence in the HIV positive population [3]. NHL and $\mathrm{HL}$ in the setting of HIV infection tend to have an aggressive clinical presentation $[4,5]$.

Prior to the widespread use of HAART, treatment for these malignancies followed the paradigm that patients could not tolerate intensive chemotherapy due to their underlying immunodeficiency. Randomized trials of standard doses of combination chemotherapy such 
as M-BACOD vs. reduced doses showed inferior results for the standard dose arm due to increased hematologic toxicity and infections [6]. However, with the widespread use of antiretroviral therapy it became possible to treat patients with more dose intensive therapy [7]. As a consequence the median survival for patients with HIV associated lymphoma has improved [8].

\section{Stem Cell Transplantation}

For patients with relapsed NHL, randomized trials have demonstrated that high dose therapy with ASCT is superior to standard dose salvage therapy in the HIV negative setting [9]. This approach has also been used in high-risk patients who are in first remission. Nonrandomized trials of this high risk group have demonstrated high rates of progression free survival (PFS) [10]. Similarly, trials of HIV negative patients with HL have shown that ASCT can provide long-term PFS for patients with relapsed disease [11]. As patients with HIV associated lymphomas now have improved hematologic reserve due to antiretroviral therapy, the use of high dose therapy with ASCT has been explored. This review will summarize these trials.

In addition, recent studies suggest that other hematologic malignancies such as acute myelogenous leukemia (AML) may have a higher incidence in the HIV infected patient. A recent study suggests a two fold increased risk of AML compared to the general HIV negative population [12]. For HIV negative patients with high risk AML or relapsed AML, generally allogeneic transplantation is utilized rather than ASCT. Hence, the question now arises about the feasibility of allogeneic transplant in the HIV infected patient with hematologic malignancies such as AML. The challenges of this approach as well as the results of recent series will be reviewed.

\section{Stem Cell Mobilization in HIV positive patients}

The first issue to be addressed in the autologous transplant patient, regardless of HIV status, is the potential for an adequate stem cell collection. Lymphoma patients have the challenge of prior exposure to myelosuppressive chemotherapy and radiotherapy. In addition, the HIV infected patient also suffers from the effects of the HIV virus on the bone marrow, and the potentially myelosuppressive effects of certain antiretroviral therapies. The HIV virus is known to alter the bone marrow microenvironment and cytokine milieu [13]. However, it does not infect the primitive stem cells [14]. In addition, antiretroviral therapy can also have positive effects on the bone marrow as manifest by improved blood counts in HIV positive patients on HAART without lymphoma [15]. In vivo studies demonstrate an increase in marrow mononuclear cells and functional improvement in progenitor cell and stem cell assays with the addition of antiretrovirals [16]. Clinical studies have found an inverse relationship between the baseline CD4+ cell count and mobilizable CD34+ cell counts [17]. However, certain antiretrovirals such as Azidothymidine (AZT) are known to be myelosuppressive and therefore, their use is contraindicated in patients prior to stem cell mobilization. Other centers also follow this practice of avoiding AZT in preparation to stem cell collection [18]. However, poor mobilization remains a challenge. In our center three out of forty one HIV positive patients failed to mobilize after chemotherapy based priming. One patient was subsequently collected with AMD 3100, a reversible inhibitor of chemokine stromal derived factor I (SDF_I) binding to CXCR4. The downregulation of SDF-I binding results in rapid egress of hematopoietic stem cells from the marrow In the other large reported series from Italy, five patients out of sixteen enrolled were mobilization failures, three after second line chemotherapy and two after cyclophosphamide [19].

Another theoretical concern with stem cell collection is whether the use of GCSF could have deleterious effects on the HIV viral load. However, studies have shown that in fact G-CSF 
caused no significant changes in HIV viral load and demonstrated the clonogenic potential of CD34+ Thy 1+ stem cells collected through apheresis from the HIV infected patients. In the studies from City of Hope and from Italy no increases in opportunistic infections or viral loads were seen during stem cell mobilization [20].

\section{Autologous Stem Cell Transplantation (ASCT)}

The initial reports of ASCT in HIV infected patients were from France in the pre HAART era [21]. A 40 year old male with HIV-NHL who was initially on zidovudine and zalcitabine for his HIV, underwent ASCT with BEAM (BCNU, Etoposide, Cytarabine, Melphalan) conditioning for relapsed disease. Of note, the antiretroviral therapy was discontinued at the time of his NHL diagnosis and resumed only as monotherapy with lamivudine five months post ASCT. The patient engrafted but his post transplant course was complicated by cytomegalovirus (CMV) viremia, atypical mycobacterium pneumonia, and intestinal cryptosporidiosis. The authors concluded that high dose therapy with ASCT was feasible in the HIV infected patient but that until better HIV therapies were available, infection would remain a significant cause of morbidity.

With the widespread use of HAART in the mid 1990's, investigators again explored ASCT in HIV positive patients. The initial series from France included fourteen patients on HAART with relapsed or refractory NHL [22]. Attempts were made to maintain patients on HAART through stem cell collection and transplant and in fact thirteen of the fourteen were able to stay on HAART throughout. The series included a variety of histologies; six with HD, 3 Burkitts type, NHL, 2 immunoblastic, NHL, 1 lymphoblastic NHL, and 1 primary effusion lymphoma. The conditioning regimens included high dose chemotherapy alone in six patients and high dose chemotherapy with total body irradiation in eight patients. Despite the use of HAART, opportunistic infections did occur. Two patients developed CMV viremia without any associated CMV disease. Of note there were no statistically significant differences in the viral load pre and post transplant for the group. Eight patients died, six from lymphoma, one from AIDS and one from a second tumor. However, the study was encouraging in that it set a platform that ASCT was feasible and with minimal infectious complications and deleterious effects on the HIV infection.

Other centers have also reported similar experiences. A larger trial from the City of Hope included twenty patients on HAART with either high risk NHL in first remission or relapsed HD or NHL [23]. All patients were on HAART. Seventeen patients received a chemotherapy based regimen of Cyclophosphamide, BCNU, Etoposide and three received a radiation based regimen of $1200 \mathrm{cGY}$ total body radiation (TBI), and cyclophosphamide, plus etoposide. All patients engrafted, though one patient had a delayed engraftment of 23 days. This was ascribed to the use of AZT as part of his antiretroviral therapy. An attempt was made to continue HAART for all patients, but only nine were able to tolerate it throughout. The remainder resumed it at a median of two months post ASCT. The CD4 counts nadired at six months post ASCT and recovered to pretransplant levels by one year. At the time of initial reporting with a median follow up of nearly 32 months, progression free survival was $85 \%$. The series was recently updated to include 28 patients [24]. Median follow-up was 41 months and PFS was 78\%. The incidence of opportunistic infections (OI) in this series was low and included PCP pneumonia in two patients not compliant with prophylaxis, CMV infection in three patients and VZV in two. No patient died of an OI.

The AIDS clinical trial group reported the results of a multicenter trial using a different preparative regimen. They used a regimen that had originally been designed for older patients in an attempt to reduce regimen related toxicity (RRT) [25]. Twenty-seven patients were enrolled on the study, ultimately twenty underwent transplant. One patient died of veno 
occlusive disease. At day 100, 53\% achieved a complete remission. Six month event free survival was $49.5 \%$. The authors emphasized that seven patients did not receive a transplant due to disease progression, and underscored the importance of patient selection i.e. chemotherapy responsive lymphoma and controlled HIV infection.

All the aforementioned studies confirmed the feasibility, tolerability and efficacy of high dose therapy and ASCT in HIV patients. In vitro studies of immune reconstitution such as interleukin 7 levels, $\mathrm{T}$ cell subsets and $\mathrm{T}$ cell $\mathrm{V}$ - Beta repertoires in a small number of HIV patients who underwent ASCT confirm the stability of immune reconstitution [26].

Investigators have also attempted to assess if the efficacy of the procedure is comparable to HIV negative patients. An EBMT series of $40 \mathrm{HIV}$ positive patients compared them to 40 HIV negative lymphoma patients matched for histology, age, disease status at ASCT and year and country of ASCT. With a median follow up of 36 months the PFS for HIV positive patients was not statistically different at $62 \%$ for HIV positive patients and $69 \%$ for HIV negative patients. The authors concluded that HIV status should not preclude patients from transplant but that infectious complications should be monitored closely [27].

\section{Allogeneic Transplantation}

Solid organ transplantation set the platform for allogeneic stem cell transplantation in that solid organ transplant patients also need chronic immunosuppression. Hence, the issues of committing an already immunocompromised individual to further immunosuppression and the concerns of interactions between antiretrovirals and immunosuppressive medications are the same. The paradigm shift in solid organ transplantation has occurred somewhat earlier than allogeneic stem cell transplantation. In fact, some organ transplant centers assert that "the HIV patient should be considered no different than any other high risk patient such as the older patient or the diabetic." [28].

\section{Immune Recovery in Allogeneic Transplant}

The literature on allogeneic transplantation in HIV positive patients is much more sparse than ASCT. Data on allogeneic transplantation in patients with other disorders suggests that the rate of immune reconstitution post transplant is dependent on factors such as the type of conditioning regimen, HLA compatibility of the donor and host, and the development of graft versus host disease (GVHD). In a transplant with an HIV negative recipient T lymphocyte recovery occurs by thirty days post transplant, though initially with predominantly CD8+ cells [29]. Recovery of CD4+ cells can take up to six months as does recovery of normal $\mathrm{T}$ cell responses to mitogens [30]. Often patients achieve a state of partial chimerism post transplant due to host- graft tolerance, not unlike that seen in solid organ allografting. Patients with non HIV mediated immunodeficiency who achieve partial chimerism with allografting may recover adequate $\mathrm{T}$ cell function. It is unknown whether the same degree of recovery would occur in the HIV infected patient.

Early reports of allogeneic transplant were in the pre HAART era. Investigators at John's Hopkins reported on a 41 year old male with HIV lymphoma who received TBI cyclophosphamde conditioning followed by allogeneic bone marrow infusion. Pre transplant he received high dose AZT and post transplant he received a lower dose. [31]. There was no significant regimen related toxicity. The patient engrafted at day +17 but ultimately died of lymphoma at day +47. At autopsy, no evidence of HIV either by culture or pcr was found in tissue specimens. This early experience underscored the feasibility of the procedure.

The EBMT published in abstract a retrospective review of 30 patients with varied hematologic malignancies who received an allograft between the years of 1987 and 2003 
[32]. A variety of conditioning and GVHD prophylaxis regimens were used. The overall incidence of Grade II-IV GVHD was 9\%. Treatment related mortality (TRM) was $46 \%$ at 100 days and $68 \%$ at 2 years. Causes of death included pulmonary toxicity in eight patients, infection in four and HIV disease in two. There was a marked difference in survival in patients transplanted after 1996 ( 4 out of 8 surviving) versus 2 out of 22 pre 1996. The authors ascribe this survival difference to the widespread use of HAART post 1996. Hence this largest experience underscores the points that both reduction of TRM and control of the HIV infection are crucial to the success of allografting.

\section{Reduced Intensity Conditioning}

Alternate conditioning strategies that allow the establishment of an allogeneic graft without fully ablative conditioning may be a more attractive strategy in the HIV patient. Studies in dogs have shown that using low dose TBI with post transplant immunosuppression can facilitate stable mixed chimerism post allografting [33]. This approach has been tried in humans. Initial studies were in older patients and those with organ dysfunction that would preclude intensive conditioning. The trial demonstrated that engraftment was possible as was clinical and molecular remissions of patients with poor risk hematologic malignancies [34].

Therefore, the use of nonmyeloablative conditioning with its sufficient anti leukemia and or anti lymphoma effects and reduced regimen related toxicity may be the optimal approach in the HIV positive patient with a hematologic malignancy. Investigators at the NCI explored this approach [35]. Two patients received a cyclophosphamide and fludarabine based conditioning regimen. HAART was held one week prior to conditioning and resumed once the patients were maintaining adequate oral intake. Cyclosporin was used for GVHD prophylaxis. One patient developed grade II acute skin GVHD which was treated with prednisone. Despite the use of stronger immunosuppression the HIV infection remained well controlled. One patient died of relapsed disease at twelve months and one remained in remission at two years of follow up. Overall the experience showed that the reduced intensity allografting is possible in the HIV infected patient and that immunosuppression can be given with HAART. Clinical protocols are ongoing at the Fred Hutchinson Cancer Center and the City of Hope using different reduced intensity conditioning regimens for HIV positive patients with varied hematologic malignancies.

\section{Conclusion}

The role of ASCT has been well established in the HIV negative setting for the treatment of relapsed or high risk lymphoma. Multiple studies have now demonstrated that ASCT in a person with underlying HIV infection is feasible and has low RRT. The transplant approach to patients with HIV lymphoma at the City of Hope is to evaluate the lymphoma and to consider the HIV as a chronic condition akin to diabetes. We therefore, consider patients with relapsed chemosensitive HIV associated NHL or HL as potential transplant candidates. In addtion, select patients with NHL in first remission and high risk features by the international prognostic index are also evaluated for ASCT. Similar to well controlled diabetes, well controlled HIV infection does not significantly increase the incidence of infections post ASCT if a program of adequate surveillance and prophylaxis is used. Future directions of study at the City of Hope are to use the platform of high dose myeloablative therapy for gene therapy. Specifically, after high dose chemotherapy patients are "rescued" with both unmodified stem cells as well as stem cells that have been transduced using a lentivirus vector containing three anti HIV RNA elements (a short hairpin RNA targeted to tat/rev, a TAR specific decoy sequence and a ribozyme targeted to CCR5) [24]. TAR or transcription activation response element is a hairpin like structure at the beginning of the 
HIV transcript which binds TAT a protein responsible for HIV RNA transcription. The TAR decoy sequence therefore, binds and sequesters TAT from viral RNA thereby blocking HIV transcription. Two patients have been transplanted utilizing this strategy and results will be presented in the near future. Allogeneic stem cell transplantation in the HIV positive patient is still in its infancy. However, some of the theoretical concerns such as pharmacokinetic interactions between HAART and immunosuppressive medication and infection risk have been allayed by the experience in solid organ transplantation in HIV positive patients. Approximately 40,000 new cases of HIV infection occur in the US annually. As these individuals age their cancer risks will continue to rise. Therefore broader application of therapies such as stem cell transplantation to the HIV infected patient with a hematologic malignancy will continue to be of importance.

\section{Acknowledgments}

Supported in part by United States Public Service Grants CA30206, CA33572 and AI 38592 and MO1 RR-43 from the General Clinical Research Center for Research Resources, National Institutes of Health, Bethesda MD.

\section{References and recommended reading}

Papers of particular interest; published within the annual period of review, have been highlighted as:

- of special interest

1. Binnet F, Lewden C, May T, et al. Malignancy-related causes of death in human immunodeficiency virus infected patients in the era of highly active antiretroviral therapy. Cancer. 2004; 10:317-324.

2. Goedert JJ, Cote TR, Virgo P, et al. Spectrum of AIDS associated malignant disorders. Lancet. 1998; 351:1833-1839. [PubMed: 9652666]

3. Biggar R, Jaffe E, Goedert J, et al. Hodgkin Lymphoma and immunodeficiency in persons with HIV/AIDS. Blood. 2006; 108:3786-3791. [PubMed: 16917006]

4. Levine AM. Acquired Immune deficiency syndrome related lymphoma; clinical aspects. Semin Oncol. 2000; 27:442-453. [PubMed: 10950371]

5. Tirelli U, Errante D, Dolcetti R, et al. Hodgkin's disease and immunodeficiency virus infectin infection; clinicopathological and virologic features of 114 patients from the Italian Cooperative Group on AIDS and Tumors. J Clin Oncol. 1995; 13:1758-1767. [PubMed: 7541452]

6. Kaplan LD, Strauss DJ, Testa MA, et al. Low dose MBACOD compared with standard dose mBACOD chemotherapy for non-Hodgkin's lymphoma associated with human immunodeficiency virus infection. N Engl J Med. 1997; 336:1641-1648. [PubMed: 9171066]

7. Noy A. Update in HIV associated lymphoma. Current Opinion in Oncology. 2004; 16:450-454. [PubMed: 15314514]

8. Lim ST, Karim R, Nathwani B, et al. AIDS related burkitts lymphoma versus diffuse large cell lymphoma in the pre highly active antiretroviral therapy (HAART) eras; significant differences in survival with standard dose chemotherapy. Journal Clin Oncol. 2005; 23:4430-4438.

9. Philip T, Guglielmi C, Hagenbeek C, et al. Autologous Bone Marrow Transplantation as compared with salvage chemotherapy in relapses of chemotherapy sensitive non-Hodgkin's lymphoma. New Engl J Med. 1995; 333:1540-45. [PubMed: 7477169]

10. Haoiun C, Lepasse B, Gisselbrecht C, et al. Benefit of autologous bone marrow transplantation over sequential chemotherapy in poor risk aggressive non-Hodgkin's lymphoma. Updated results of the LNH37-2 Groupe d'etude des lymphomas de l'adulte. J Clin Oncol. 1999; 13:1131-7.

11. Linch DC, Winfield D, Goldstone AH, et al. Dose intensification with autologous bone marrow transplantation in relapsed and resistant Hodgkin's disease: results of a BNLI randomized trial. Lancet. 1993; 341:1051-54. [PubMed: 8096958] 
12. Sutton L, Guenel P, Tanguy ML, et al. Acute Myeloid Leukemia in human immunodeficiency virus infected adults; epidemiology, treatment feasibility, and outcome. Br J Haematol. 2001; 112:900. [PubMed: 11298584]

13. Koka PS, Jameson BD, Brooks DG, et al. Human Immunodeficiency virus type 1 induced hematopoietic inhibition is independent of productive infection of progenitor cells in vivo. J Virol. 1999; 73:9089-9097. [PubMed: 10516015]

14. Weichold F, Zella D, Barabitskaja O, et al. Neither Human Immunodeficiency Virus (HIV-1) nor (HIV-2) infects most primitive human hematopoietic stem cells as assessed in long term bone marrow cultures. Blood. 198(91):907-915.

15. Huang SS, Barobour JD, Deeks SG, et al. Reversal of human immunodeficiency virus type 1 associated hematosuppression by effective antiretroviral therapy. Clin Infect Dis. 2000; 30:504510. [PubMed: 10722435]

16. Isgro A, Mezzaroma I, Aiuti A, et al. Recovery of hematopoietic activity in bone marrow from human immunodeficiency virus type- 1 infected patients during highly active antiretroviral therapy. AIDS Res Human Retroviruses. 2000; 16:1471. [PubMed: 11054260]

17. Schooley RT, Mladenovic J, Sevin A, et al. Reduced Mobilization of CD34+stem cells in advanced human immunodeficiency virus type-1 disease. J Infect Dis. 2000; 181:148-157. [PubMed: 10608761]

18. Serrano D, Carrion R, Balsalobre P. HIV associated lymphoma successfully treated with peripheral blood stem cell transplantation. Exp Hematol. 2005; 33:487-94. [PubMed: 15781340]

19. Re A, Cattaneio C, Michielli M, et al. High Dose Therapy and Autologous Peripheral blood Stem cell transplantation as salvage treatment for HIV associated lymphoma in patients receiving highly active antiretroviral treatment. J Clin Oncol. 2003; 23:4423-4427. [PubMed: 14581441]

20. Krishnan A, Molina, Zaia J, et al. Autologous Stem Cell Transplantation for HIV associated Lymphoma. Blood. 2001; 98:3857-3859. [PubMed: 11739198]

21. Gabarrre J, Leblond V, Sutton L, et al. Autologous bone Marrow transplantation in relapsed HIV related Non-Hodgkin's lymphoma. Bone Marrow Transplantation. 1996; 18:1195-1197. [PubMed: 8971396]

22. Gabarre J, Choquet S, Azar N, et al. High Dose Chemotherapy with autologous stem cell transplant for HIV associated lymphoma: A large single center report on 14 patients. Blood. 2001; 98:a2092.

23. Krishnan A, Molina A, Zaia J, et al. Durable Remissions with autologous stem cell transplantation for high risk HIV associated lymphomas. Blood. 2005; 105:874-878K. [PubMed: 15388574]

24. Krishnan A, Zaia J, Rossi J, et al. Autologous Stem Cell Transplantation (ASCT) for AIDS related lymphomas and the potential role of HIV resistant stem cells. Blood. 2006; 108:491.

25. Spitzer T, Ambinder R, Lee J, et al. Dose Reduced Busulfan, Cyclophosphamide and Autologous Stem Cell Transplantation for Human Immunodeficiency Virus Associated Lymphoma: Aids Malignancy Consortium Study 020. Biol Blood and Marrow Transplantation. 2000; 14:59-66.

26. Benicchi T, Ghidini C, Re A, et al. T cell immune reconstitution after hematopoietic stem cell transplantation for HIV associated lymphoma. Transplantation. 2005; 80:673-682. [PubMed: 16177644]

27. Dies-Martin J, Balsalobre P, Re A, et al. Comparable Survival between HIV + and HIV Hodgkin's Lymphoma and Non-Hodgkin's Lymphoma patients undergoing an autologous peripheral blood stem cell transplantation. A Retrospective analysis of the EBMT Lymphoma working party. Blood. 2007; 110:24.

28•. Persad G, Little R, Grady C. Including Persons with HIV infection in Cancer Clinical Trials. J Clin Oncol. 2008; 26:1027-1032. This editorial reviews the concerns about why HIV patients have been previously excluded from cancer clinical trials and makes the points that many of those prior concerns are no longer valid. [PubMed: 18309938]

29. Keever CA, Small TN, Flomenberg N, et al. Immune Reconstitution following bone marrow transplantation: Comparison of recipients of $\mathrm{T}$ cell depleted marrow with recipients of conventional marrow grafts. Blood. 1989; 73:1340-1350. [PubMed: 2649174]

30. Gratama JW, Fibbe WE, Visser JW, et al. CD3+,4+ and or 8+ T cells and CD3+4-,8- T cells repopulate at different rates after allogeneic bone marrow transplantation. Bone Marrow Transplantation. 1989; 4:291-296. [PubMed: 2543471] 
31. Holldand K, Saral R, Rossi J, et al. Allogeneic Bone Marrow Transplantation, Zidovudine and Human Immunodeficiency Virus Type 1 infection. Annal Internal Medicine. 1989; 111:973-981.

32. Gupta V, Tomblyn M, Pederson T, et al. Allogeneic Hematopoietic Stem Cell Transplantation in HIV positive patients with malignant and non malignant disorders: a report from the center for international blood and marrow transplant research (CIBMTR). Biol of Blood and Marrow Transplantation. 2007; 13:5-6. This is the largest review of allogeneic transplant in HIV positive patients. It is retrospective and multicenter. In addition as many patients were in the pre HAART era the results may not reflect the potential of allogeneic transplant with the current therapy of HIV.

33. Storb R, Raff RF, Appelbaum FR, et al. DLA Identical bone marrow grafts after low dose total body irradiation. The effect of caninc recombinant hematopoietic growth factors. Blood. 1994; 84:3558-3566. [PubMed: 7524741]

34. Corradini P, Tarella C, Olivieri A, et al. Reduced intensity conditioning followed by allografting of hematopoietic cells can produce clinical and molecular remissions in patients with poor- risk hematologic malignancies. Blood. 2002; 99:75-82. [PubMed: 11756155]

35. Kang E, de Witte M, Malech H, et al. Nonmyeloablative conditioning followed by transplantation of genetically modified HLA matched peripheral blood progenitor cells for hematologic malignancies in patients with acquired immunodeficiency infection. Blood. 2002; 99:698-701. [PubMed: 11781257] 